\title{
Recurrent Otorrhea in A Child: Early Sign of Temporal Bone Langerhans Cell Histiocytosis
}

\author{
Mohd Zulkefli $N^{a}$, Goh BS ${ }^{a, b}$, Amri $F^{c}$, Tan $G C^{c}$ \\ aDepartment of Otorhinolaryngology-Head and Neck Surgery, Faculty of Medicine, Universiti Kebangsaan \\ Malaysia Medical Centre (UKMMC), Kuala Lumpur, Malaysia. \\ bInstitute of Ear, Hearing and Speech (Institute-HEARS), Jalan Temerloh, Universiti Kebangsaan Malaysia \\ (UKM), Kuala Lumpur, Malaysia. \\ 'Department of Pathology, Universiti Kebangsaan Malaysia Medical Centre (UKMMC), Kuala Lumpur, \\ Malaysia.
}

\begin{abstract}
Langerhans cell histiocytosis $(\mathrm{LCH})$ is a rare disease of dendritic origin with uncertain etiology, with predilection in children. A 1-year 6-month girl with history of recurrent otorrhea, presented with mass in the ear canal and computed tomography imaging showed expansile lytic bony lesion in right mastoid with eroded ossicles. Subsequently, histopathological examination confirmed the diagnosis of LCH and chemotherapy was successfully commenced with complete resolution of symptoms. LCH with ear involvement are usually mistaken as other acute or chronic ear disease such as chronic otitis media or mastoiditis. Hence, in children with recurrent otorrhea with ear canal mass, with or without facial nerve palsy, LCH should be considered as one of the differential diagnosis.
\end{abstract}

KEYWORDS: Langerhans cell histiocytosis, children, otorrhea, temporal bone.

\section{INTRODUCTION}

Langerhans cell histiocytosis $(\mathrm{LCH})$ is a rare disease affecting children age 1 to 3 years old with an annual incidence of 2.4 - 5.6 cases per million children. ${ }^{1,2}$ The most accepted theory of LCH etiology is a clonal proliferation of Langerhans cells, which are the mononuclear cell of bone-marrow origin that belongs to the dendritic cell family and potent antigen-presenting cell. ${ }^{2,3}$ It is classified into a single organ system (unifocal or multifocal) or multi-organs system ( 2 or more organs or systems) according to Histiocyte Society and World Health Organization (WHO).,4 $\mathrm{LCH}$ of the head and neck accounts to $50-80 \%$ of all cases, with temporal bone involvement around 15-60\%.,6 Temporal bone LCH has a similar presentation to other common otology diseases such as mastoiditis, recurrent otitis media, or otitis externa. The presence of proliferating Langerhans dendritic cells, Birbeck granules, and

Corresponding Author:

Prof. Dr. Goh Bee See

Department of Otorhinolaryngology- Head \& Neck Surgery, Universiti Kebangsaan Malaysia Medical Centre, Jalan Yaacob Latif, Bandar Tun Razak,

56000 Kuala Lumpur, Malaysia.

Tel No : +603-91456045

Email : beesee@ppukm.ukm.edu.my positive immunohistochemical staining for CD1a and S100 are pivotal to diagnose LCH. Here we report this rare case to raise awareness, timely diagnosis, and prompt treatment of the disease.

\section{CASE REPORT}

A 1-year 6-month old girl presented with a prolonged cough for 1 month and was treated for left upper lobe pneumonia. During follow up, a repeated chest radiograph showed persistent left upper lobe homogenous opacities with a mediastinal mass. Further history revealed right neck swelling of unsure duration and intractable right otorrhea since the age of 3 months. She has lost $1.5 \mathrm{~kg}$ within a month with night sweats but without any tuberculosis (TB) contact and no dyspnea.

On examination, the child was not in respiratory distress and the facial nerve was intact. There were multiple sub-centimeter lymph nodes palpable at right neck level II and presence of distended veins at abdomen and chest with hepatomegaly. On otoscopy, noted a lobulated mass occupying the whole right ear canal with non-foul smelling whitish discharge, obscuring the view of the tympanic membrane. 
Serial chest radiographs showed persistent homogenous opacities of the left upper lobe with a widening of mediastinum. Computed tomography (CT) scan of thorax reported a locally aggressive anterior mediastinal mass with left bronchial compression causing left upper lobe collapse and mass effect onto the trachea as well as superior vena cava (SVC) obstruction. High-resolution CT temporal bone showed features of an expansile lytic bony lesion involving right mastoid air cells and middle ear cavity with erosion of part of the ossicles and mastoid portion of the right facial nerve canal (Figure 1). Magnetic radiograph imaging (MRI) of the whole-body reported that the anterior mediastinal mass likely arises from thymus with compression features, mass in the right ear canal, and mastoid with evidence of skull base erosion without intracranial extension. There were also osseus (left humerus, bilateral metacarpal bones, and right iliac wing) and hepatobiliary involvement.

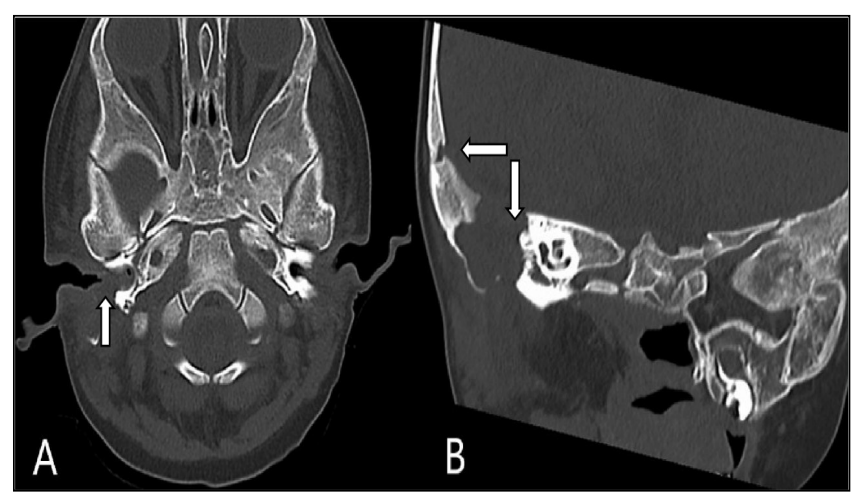

Figure 1: (A) Axial view showing expansile lytic right intratemporal bony lesion involving mastoid air cells (white arrow), posterior wall of the external auditory canal and middle ear cavity. (B) Coronal view showing bony erosion "punched-out lesion" at lateral and posterior right temporal squama and tegmen tympani (white arrow). The erosive changes also include a long process of incus and the mastoid segment of the right facial nerve.

Histopathological examination of the right ear canal lesion (Figure 2 ) showed that the neoplastic cells are positive for S100 and CD1a; and negative for LCA, CD3, and CD79a. Hence, this patient was diagnosed with multisystem $\mathrm{LCH}$ with organ involvement (temporal bone with the risk of intracranial involvement, thymus, liver, and osseus: sternum, humerus, metacarpal, and iliac).

She was referred to a paediatric oncologist and completed 2 cycles of LCH protocol (vinblastine with oral steroids) and continuation chemotherapy with vinblastine (52 weeks). Currently, she is in remission with no sequelae. A surveillance MRI is scheduled to monitor her disease.

\section{DISCUSSION}

$\mathrm{LCH}$ is more commonly seen in boys than females in a ratio of $2: 1 .^{6,7}$ There is no specific clinical manifestation that is pathognomonic for temporal bone $\mathrm{LCH}$, it depends on the site and extension of the disease. Our case presented with recurrent refractory otorrhea and aural polyp, which correlate with findings of Fernandez et al and Skoulakis et al. However, recent literature reported most common manifestations are temporal bone lesion or mastoid swelling (74.1\%), periauricular swelling (44.4\%), otorrhea (33.3\%), otalgia (22.2\%) and conductive hearing loss. ${ }^{2,5-8}$ Other symptoms such as ear canal polyp, sensorineural hearing loss, vertigo, and facial nerve palsy are rare. ${ }^{7,9}$ Saliba et al postulated that the bony labyrinth is rarely susceptible to erosion in $\mathrm{LCH}$ and Postovsky et al stated that inert growth of $\mathrm{LCH}$ tends to not infiltrate neural tissue. This markedly different from other diseases of mastoid or middle ears such as cholesteatoma or chronic otitis media.

All authors unanimously agreed that CT scan is the investigation of choice to detect bony erosion in $\mathrm{LCH}$ while MRI can depict the soft tissue extension and relationship with adjacent soft tissue structures. The characteristic feature in CT scan includes diffusely destructive lytic bony lesions at the ear canal, mastoid process, posterior and lateral semicircular canal, but rarely involve ossicular chain, facial canal, and petrous apex. ${ }^{3,5-7,10} \mathrm{~A}$ typical "punched-out lesion" at the ear canal and squamous part of the temporal bone is characteristic as per this case. $^{5}$ Zheng et al hypothesized that the ossicular chain is resistant to granulation tissue of $\mathrm{LCH}$ and ossicles destruction likely contributed by existing chronic otitis media.

Diagnosis of ear involvement $\mathrm{LCH}$ is challenging to untrained eyes as it has very similar presentations to other more common ear diseases. Histopathological examination and positive immunohistochemical staining to S100 and CD1a is paramount for diagnosis of $\mathrm{LCH}$ and typically performed as they are specific and cost-effective. ${ }^{7}$ Identification of Birbeck granules aid confirmation of $\mathrm{LCH}$ but not routinely 


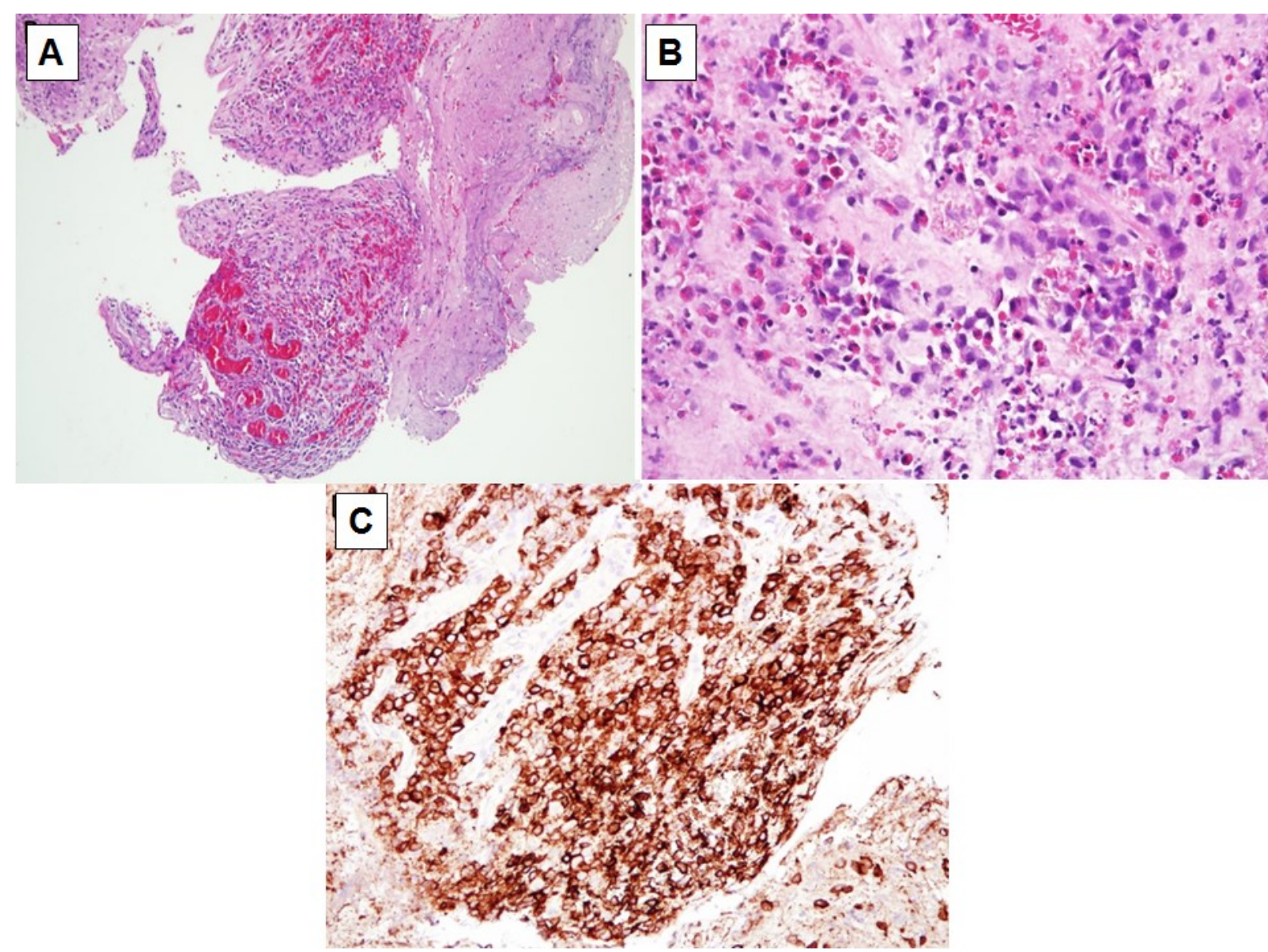

Figure 2: (A) Tumour composed of neoplastic cells admixed with inflammatory cells and blood vessels (H\&E, 100x). (B) The neoplastic cells exhibit oval to bean-shaped, grooved, lobed nuclei, fine chromatin, inconspicuous nucleoli, moderate eosinophilic cytoplasm. The background cells consist of scattered neutrophils, lymphocytes, and dense eosinophils forming eosinophilic microabscesses (H\&E, 400x). (C) The neoplastic cells are positive toward CD1a immunohistochemistry (200x).

done as they may be absent. Hence, early imaging is recommended, and obtaining tissue biopsy is a priority.

The treatment options for LCH include surgery, chemotherapy, radiotherapy, local steroids injection, or a combination of them, depending on the extension of $\mathrm{LCH}^{6,7}$ Histiocyte Society established that combination treatment with vinblastine and prednisolone are effective with minimal side effects; and are the standard therapy for multisystem LCH. ${ }^{2,3}$ This is supported by recent literature where chemotherapy is preferred especially for multisystem LCH. Complete surgical excision or intralesional steroid injection is sufficient in the accessible, localized lesion. However, in temporal bone LCH, mastoidectomy may be performed for diagnostic and therapeutic purposes. In our case, tissue biopsy was obtained from ear canal polyp. Radiotherapy is no longer recommended as an initial treatment but as an adjuvant in relapse or residual diseases.

\section{CONCLUSION}

Our experience from this rare case raised the importance of early referral to ENT surgeon in case of refractory otorrhea or aural polyp. LCH of the temporal bone may occur alone or as part of multisystem involvement. Therefore, an examination of other systems must be carried out if $\mathrm{LCH}$ is diagnosed. A lytic "punched-out lesion" of temporal bone or skull is characteristic of $\mathrm{LCH}$. Prognosis of multisystem LCH depends on the child age and disease extension, therefore multidisciplinary effort is pertinent to recognize and treat LCH early for a better outcome.

\section{REFERENCES}

1. Skoulakis CE, Drivas El, Papadakis CE, et al. Langerhans cell histiocytosis presented as bilateral otitis media and mastoiditis. 50:5.

2. Schmidt S, Eich G, Geoffray A, et al. 
Extraosseous Langerhans Cell Histiocytosis in Children. RadioGraphics. 2008; 28:707-726.

3. Grana N. Langerhans Cell Histiocytosis. Cancer Control. 2014; 21:328-334.

4. Tantiwongkosi B, Goske MJ, Steele M. Congenital solid neck mass: a unique presentation of Langerhans cell histiocytosis. Pediatric Radiology. 2008; 38:575-578.

5. Zheng H, Xia Z, Cao W, et al. Pediatric Langerhans cell histiocytosis of the temporal bone: clinical and imaging studies of 27 cases. World J Surg Onc. 2018; 16:72.

6. Yadav V, Kaushal D, Gugliani A. Pediatric temporal bone langerhans cell histiocytosis: report of 2 cases with review of literature. IJMBOA. 2019; 4:185-187.

7. Saliba I, Sidani K, El Fata F, et al. Langerhans' cell histiocytosis of the temporal bone in children. International Journal of Pediatric Otorhinolaryngology. 2008; 72:775-786.

8. Modest MC, Garcia JJ, Arndt CS, et al. Langerhans cell histiocytosis of the temporal bone: A review of 29 cases at a single center. The Laryngoscope. 2016; 126:1899-1904.

9. Postovsky S, Daitzchman M, Dale A, et al. Unusual presentation of mastoid eosinophilic granuloma in a young patient. Pediatric Hematology and Oncology. 2001; 18:283-289.

10. Fernández-Latorre $F$, Menor-Serrano F, AlonsoCharterina $S$, et al. Langerhans' Cell Histiocytosis of the Temporal Bone in Pediatric Patients. American Journal of Roentgenology. 2000; 174:217-221. 